Discussion Paper Series No. 178

\title{
Sustainability or ruin of a common resource economy with random jump

$\begin{array}{ll}\text { FUJISAKI Masatoshi } & \text { University of Hyogo } \\ \text { KATAYAMA Seiichi } & \text { Kobe University } \\ \text { OHTA Hiroshi } & \text { Kobe University }\end{array}$

December 2005

Discussion papers are a series of manuscripts in their draft form. They are not intended for circulation or distribution except as indicated by the author. For that reason Discussion Papers may not be quoted, reproduced or distributed without the written consent of the author. 


\title{
Sustainability or ruin of a common resource economy with random jump
}

\author{
FUJISAKI Masatoshi, University of Hyogo, \\ KATAYAMA Seiichi, Kobe University, RIEB \\ OHTA Hiroshi, Kobe University, GSICS
}

2005.11.22.

\begin{abstract}
We consider a common resource economy in which agents exploit the common resource, and use it to produce goods and consume the goods produced. Also the agents can invest in private and productive capital. The resource extracted from the common resource is non-renewable and the common pool is under unceratinty in the sense that it could have a sudden increase or decrease in the course of extraction. In this model we shall explore the probability of sustainability or ruin of the economy. We compare and discuss the optimal extraction rules and the probabilities under cooperative and non-cooperative regimes.
\end{abstract}

Keywords: common property resource, private capital accumulation, pure jump process, exhaustion probability, HJB (Hamilton-Jacobi-Bellman) equation

JEL Classification: Q32

Corresponding author: Seiichi Katayama, RIEB, Kobe University, 2 Rokkodai, Nada-ku, Kobe, Japan, Fax: +81 788037012

E-mail:katayama@rieb.kobe-u.ac.jp 


\section{Introduction}

On the plannet Earth the amount of natural resources is limited and their endowments are unevenly located. However, resources held by some limited number of countries are of free access under suitable economic conditions. From the consumers' point of view natural resource is nothing but a common property.

It is well known that when agents have free access to a common property resource, they tend to over-exploit the resource stock. The size of the resource stock thus declines at a much faster rate than the socially optimal rate. In most of the models of common property resources, the typical assumptions are that there are no other stocks than resources, such as private capital, and that the extracted resources are directly consumed .

To shed light on the alternative capital asset than resources, Long and Katayama (2002) have presented a model of exploitation of a common property resource, when agents can also invest in private and productive capital. The resource extracted from a common pool is non-renewable in the model. They investigate the pattern of consumption and capital accumulation. In the present paper, we try to extend their result to the case where a common pool is under uncertainty in the sense that it could have a sudden increase or decrease in the course of extraction.

The extension is quite natural when we see the present state of international crude oil market. Some oil producing countries have encountered the technological difficulties of extraction and /or social hazards. Also the past history of oil shows that unexpected exploration of new oil reserves occasionally happened. Even so, we know that the total reserve in the earth is limited, and it is expected that the resource is finally exhaustible. As a remedy, people can accumulate capital for substituting the exhaustible resource and prolong the ultimate depletion of resource stock.

To take into account this consideration to our discussion, we present a model of uncertainty on the available reserve of resource and build in a physical capital as substitute for the exhaustible resource. Speciffically, we build a model with a random jump in the stock of the resource. Fujisaki, Katayama and Ohta (2005) have already discussed whether there is an optimal solution to this type of model.

One of the main issues in the economy of this sort is whehter it could be sustainable or reaching to the ruin situation where it would have hit the minimum boundary of the resource reserve and the economy would cease to be active. It is interesting to see how high such probability is and which factor affects the probability. If the economy could be in destruction, which probability of ruin is higher under coopreative regime or under non-cooperative regime? Thus, we derive and compare the probabilities of ruin in both regimes. As far as the authors have known, there is no existing literature discussing sustainability and ruin of resource economy in probability sense as in this paper. The pattern of the resource extraction would be affected by the uncertainty. We obtain the optimal resource extraction rule, and ask how this rule is affected by the jump process in resource reserve and between the co-operative and non-cooperative regimes.

The structure of the paper is as follows. The next section develops the model. In the section 2 the cooperative outcome is stated. In the section 3 exhaustion and sustainable probabilities are derived. The non-cooperative outcome follows in the section 4 , and the comparison with cooperative outcome is made in terms of the ruin 
probability and the extarction rates in the section 5. The final section concludes the paper.

\section{The Model}

The basic framework of the model is same as Long and Katayama (2002).

There are $n$ identical agents having common access to a stock of nonrenewable natural resource, denoted by $S(t)$. Each agent $i$ also owns a private capital stock $K_{i}(t)$. Agent $i$ extracts the amount $R_{i}(t)$ of the common resource stock $(i=1, \ldots, n)$. Extraction is costless. Total extraction in the economy at time $t$ is $R(t)=\sum_{i=1}^{n} R_{i}(t)$, and the reserve depletes according to

$$
\dot{S}(t)=-R(t)
$$

if it is not subject to any uncertainty.

However, the reserve may be augmented or damaged several times in the finite horizon and the reserve size is affected by those jumps in magnitude. Let us consider a probability space $\left(\Omega, \mathcal{F}, P, \mathbf{F}=\left\{\mathcal{F}_{t}\right\}\right)$ satisfying the usual conditions and the jump process is given on this basis. The jump process takes the form $d J(t)$, and the resource stock is governed by the following stochastic differential equation (in short, $\mathrm{SDE})$

$$
d S(t)=d J(t)-R(t) d t
$$

The stok level at time $t$ is

$$
S(t)=S_{0}+J(t)-\int_{0}^{t} R(s) d s,
$$

where $J(t)$ is a pure jump process given by

$$
J(t)=\int_{0}^{t} \int_{\mathbf{R} \backslash\{0\}} S(s-) \cdot z N(d s, d z) .
$$

$N(\cdot)$ is a Poisson Random measure with intensity

$$
E[N(A)]=\lambda \int_{A} \sigma(z) d t d z
$$

$A \in B\left(\mathbf{R}_{+} \times \mathbf{R}\right)$, a Borel set and $\lambda>0 . \lambda \sigma(z) d z$ is called Lévy measure and we assume that

$$
\int_{-\infty}^{\infty}|z|^{2} \wedge 1 \sigma(z) d z<\infty .
$$

The Lévy measure expresses the possible jump size and $\lambda$ is the average number of jumps to occur during unit time interval. This rather technical assumption exculudes such cases that the jumps occur intensively in any finite intervals and/or the sizes are extremely large.

Assume that the extracted resource cannot be directly consumed. Instead, agent $i$ uses $R_{i}$ as an input, which, in combination with his labor input and his privately 
owned capital stock $K_{i}$ yields an output $Y_{i}$ of final good. For simplicity we choose a measurement unit by fixing the labor input to unity. Therfore, agent $i$ 's production function is

$$
Y_{i}=R_{i}^{1-\beta} K_{i}^{\alpha},
$$

where $0<\alpha, \beta<1$. Agent $i$ consumes $C_{i}(t)$, and the remaining quantity is invested to accumulate his physical capital. The rate of accumulation of the privately owned capital stock is thus

$$
d K_{i}(t)=\left(R_{i}^{1-\beta} K_{i}^{\alpha}-C_{i}\right) d t
$$

Each individual utility is increasing in consumption $C_{i}(t)$ :

$$
U_{i}=(1-\gamma)^{-1} C_{i}^{1-\gamma},
$$

where $0<\gamma<1$. Each agent wishes to maximize the integral of the stream of discounted utility

$$
\max \int_{0}^{\infty}(1-\gamma)^{-1} C_{i}^{1-\gamma} e^{-\rho t} d t
$$

subject to (1.1) and (1.3), and the initial conditions

$$
\begin{gathered}
S(0)=S_{0}, \\
K_{i}(0)=K_{i 0} .
\end{gathered}
$$

$\gamma$ is the elasticity of marginal utility, and for mathematical simplicity as in Long and Katayama (2002) assume that $\gamma=\alpha$.

\section{The cooperative outcome}

If the agents cooperate each other, they will collectively seek to maximize the same level of their welfare. They will choose the rate of extraction per agent $R_{h}$ and consumption per agent $C_{h}$ to maximize

$$
\max \int_{0}^{\infty}(1-\alpha)^{-1} C_{h}^{1-\alpha} e^{-\rho t} d t
$$

subject to

$$
\left\{\begin{array}{l}
d K_{h}(t)=\left(R_{h}(t)^{1-\beta} K_{h}(t)^{\alpha}-C_{h}(t)\right) d t \\
d S(t)=d J(t)-n R_{h}(t) d t
\end{array}\right.
$$

and the boundary conditions

$$
\begin{aligned}
S(0) & =S>a>0, K_{h}(0)=K_{h} \geq 0 . \\
P\left(\lim _{t \rightarrow \infty} S(t)<\infty\right) & =1, \lim _{t \rightarrow \infty} K_{h}(t) \geq 0 .
\end{aligned}
$$

For the resource economy to be analytically meaningful, the boundary condition in the future is introduced so that the resource stock in any event should be finite. 
Define the value function $V\left(S, K_{h}\right)$ for this maximization problem by

$$
V\left(S, K_{h}\right)=\max _{C_{h}, R_{h} \geq 0} E\left[\int_{0}^{\tau}(1-\alpha)^{-1} C_{h}^{1-\alpha} e^{-\rho t} d t+g\left(S(\tau), K_{h}(\tau)\right) e^{-\rho \tau}\right]
$$

where $\rho>0$ is a discount factor, $g$ is a given function which represents the evaluation of remaining stocks, $0<\alpha, \beta<1$. $\tau$ is the first exit time from $D$,

$$
\tau=\inf \left\{t>0 ;\left(S(t), K_{h}(t)\right) \notin D\right\},=\infty \text { if }\{\cdot\}=\phi,
$$

where $D=\{(S, K) ; S>a, K>0\}$ and $a$ is a positive constant. Here it is assumed that the whole system collapses when the economy reaches to $\tau$, and that it is necessary for the economy to keep the minimum sustainable level $a$ of the resource stock. This assumption is innnocuous.

It is known (see e.g. Kushner and Dupuis (2001)) that this optimization problem is equivalent to the following Hamilton-Jacobi-Bellman equation;

$$
\rho V\left(S, K_{h}\right)=\max _{C_{h}, R_{h}>0}\left\{\begin{array}{l}
(1-\alpha)^{-1} C_{h}^{1-\alpha}+V_{K_{h}}\left(R_{h}^{1-\beta} K_{h}^{\alpha}-C_{h}\right) \\
+\lambda \int_{\mathbf{R}}\left\{V\left(S+S z, K_{h}\right)-V\left(S, K_{h}\right)\right\} \sigma(z) d z+V_{S}\left(-n R_{h}\right)
\end{array}\right\}
$$

for $\left(S, K_{h}\right) \in D$, and

$$
V\left(S, K_{h}\right)=g\left(S, K_{h}\right) \quad \text { for } \quad\left(S, K_{h}\right) \in \partial D,
$$

where $V_{K_{h}}=\partial V / \partial K_{h}$ and $V_{S}=\partial V / \partial S$.

The first order condition for maximization are

$$
\left\{\begin{array}{r}
C_{h}^{-\alpha}-V_{K_{h}}=0 \\
(1-\beta) R_{h}^{-\beta} K_{h}^{\alpha} V_{K_{h}}-n V_{S}=0
\end{array}\right.
$$

They turn to be

$$
C_{h}=\left(V_{K_{h}}\right)^{-1 / \alpha}
$$

and

$$
R_{h}=\left\{\frac{n V_{S}}{(1-\beta) V_{K_{h}} K_{h}^{\alpha}}\right\}^{-1 / \beta}
$$

Substituting these conditions into Eq.(2.3), we obtain the partial differential equation for $V\left(S, K_{h}\right)$. As Long and Katayama (2002) have indicated, the solution to the differential equation is not simple. Instead of solving it directly we take the same solution as the one used by them. Assume that the partial differential equation has a simple solution

$$
V\left(S, K_{h}\right)=A K_{h}^{1-\alpha}+B S^{1-\beta}
$$


where $A$ and $B$ are positive constants to be determined. Then Eq.(2.3) becomes

$$
\begin{aligned}
& \rho\left(A K_{h}^{1-\alpha}+\right.\left.B S^{1-\beta}\right)=(1-\alpha)^{-1}\left(V_{K_{h}}\right)^{1-1 / \alpha}+V_{K_{h}}\left\{R_{h}^{1-\beta} K_{h}^{\alpha}-\left(V_{K_{h}}\right)^{-1 / \alpha}\right\} \\
&-n R_{h} V_{S}+\lambda \int\left\{V\left(S+S z, K_{h}\right)-V\left(S, K_{h}\right)\right\} \sigma(z) d z \\
&= \frac{\alpha}{1-\alpha}\left\{(1-\alpha) A K_{h}^{-\alpha}\right\}^{1-1 / \alpha}+R_{h}^{1-\beta} K_{h}^{\alpha}\left\{(1-\alpha) A K_{h}^{-\alpha}\right\} \\
&- n R_{h}(1-\beta) B S^{-\beta}+\lambda B S^{1-\beta} \int\left\{(1+z)^{1-\beta}-1\right\} \sigma(z) d z \\
&=\alpha(1-\alpha)^{-1 / \alpha} A^{1-1 / \alpha} K_{h}^{1-\alpha}+\left\{\beta(n B)^{1-1 / \beta}[(1-\alpha) A]^{1 / \beta}+\lambda B d(\beta, \sigma)\right\} S^{1-\beta},
\end{aligned}
$$

where $d(\beta, \sigma)$ is given by the following formula.

$$
d(\beta, \sigma)=\int\left\{(1+z)^{1-\beta}-1\right\} \sigma(z) d z .
$$

For this equation to hold for all $K_{h}>0$ and $S>0$, it is necessary that the following conditions are satisfied;

$$
\left\{\begin{array}{l}
\rho A=\alpha(1-\alpha)^{-1 / \alpha} A^{1-1 / \alpha} \\
\rho B=\beta(n B)^{1-1 / \beta}[(1-\alpha) A]^{1 / \beta}+\lambda B d(\beta, \sigma) .
\end{array}\right.
$$

Assume that

$$
\delta \equiv \rho-\lambda d(\beta, \sigma)>0
$$

Therefore, we deduce

$$
A=\left(\frac{\alpha}{\rho}\right)^{\alpha}(1-\alpha)^{-1}
$$

and

$$
B=\left(\frac{\beta}{\delta}\right)^{\beta} n^{\beta-1}\left(\frac{\alpha}{\rho}\right)^{\alpha} .
$$

From these results, we can obtain the following.

Proposition 2.1 Assume that $g$ in (2.2) is of the form

$$
g(S, K)=A K^{1-\alpha}+B S^{1-\beta},
$$

where $A$ and $B$ are defined by the above formulas, then the optimal consumption $C_{h}$ and the optimal resource extraction $R_{h}$ are given by

$$
\left\{\begin{array}{l}
C_{h}=\left(\frac{\rho}{\alpha}\right) K_{h} \\
R_{h}=\left(\frac{\delta}{\beta}\right)\left(\frac{S}{n}\right)
\end{array}\right.
$$


respectively. Moreover, the corresponding optimal trajectory of two stocks $\left(K_{h}, S\right)$ are given by the following differential equations;

$$
\left\{\begin{array}{l}
d K_{h}(t)=\left\{\left(\frac{\delta S(t)}{n \beta}\right)^{1-\beta} K_{h}(t)^{\alpha}-\left(\frac{\rho}{\alpha}\right) K_{h}(t)\right\} d t \\
d S(t)=d J(t)-\left(\frac{\delta}{\beta}\right) S(t) d t
\end{array}\right.
$$

with initial (boundary) conditions $K_{h}(0)=K_{0}>0$ and $S(0)=S_{0}>a>0$.

The phase diagram for this system in the $\left(K_{h}, S\right)$ plane is almost the same as Long and Katayama (2002) or Fujisaki, Ibuki and Katayama (2004). In fact, it is clear that if the jumps occur in the negative direction which could happen in such case as sudden destruction of the resource stock, then both the resource stock $S$ and capital stock $K_{h}$ will eventually converges to the boundary $(0, a)$.

Remark 2.1 It is easy to see that the assumption (A.1) is satisfied if we choose $\rho$ and $\lambda$ so that $d(\beta, \sigma)<\rho / \lambda$. The economic meaning is that the average size and the number of jumps are restricted not to be so large.

Remark 2.2 The optimal consumption rule (2.4) is independent of the stock of the resource, and as (Long and Katayama(2002)) shows, the extraction by agent $h$ depends only on the resource stock per head, $S / n$. Notice that Long and Katayama (2002) has derived the optimal extraction function as $R_{h}=[\rho / \beta] \times[S / n]$ in the absence of jumps in resource size. Our result is obtained by replacing $\rho$ with $\delta=$ $\rho-\lambda d(\beta, \sigma)$. If $d(\beta, \sigma) \geq 0$, then the optimal extraction is revealed to be less under jumps than without them for the same level of resource stock. Moreover as $\delta$ is decreasing in $\lambda$, the optimal extraction decreases as the resource jumps more frequently. It is the way for economic agents to react more cautiously to cope with the uncertainty. However, the more cautious behavior is not applied to consumption, since the optimal level $C_{h}$ is shown to be the same as in Long and Katayama (2002).

\section{Sustainablility and ruin probabilities}

In this paragraph, we will calculate the exhaustion probability of the stock process. Using the optimal resource extraction rules (2.4) and optimal trajectories (2.5), the stock process is given by the following formula;

$$
S(t)=S_{0}+J(t)-\int_{0}^{t} \eta S(s) d s,
$$

where $J$ is a pure jump process given by (1.2) and we put $\eta=\delta / \beta$. Note that (3.1) is written in terms of the following stochastic differential equation, so called stochastic exponential;

$$
d S(t)=S(t-) d Y(t), S(0)=S_{0}
$$


where $Y$ is Lévy process given by

$$
Y(t)=\int_{0}^{t} \int_{\mathbf{R} \backslash\{0\}} z N(d s, d z)-\eta t .
$$

We will need the following assumptions.

$$
\inf \{\Delta Y(t), t>0\}>-1 \text { (a.s.), }
$$

where $\Delta Y(t) \equiv Y(t)-Y(t-)$. Otherwise, $S(t)$ may be negative (see Remark 3.1 (1)). As it can be seen from (3.2), (A.2) implies that the jump size should not be too large for $S(t) \geq 0$. In the following, assume that the support of the measure $\sigma(z) d z$ is included in the interval $(-1, \infty)$ (cf. Remark 2.1). Then it is well known (see Applebaum (2004)) that the unique solution of Eq.(3.2) is represented as follows;

$$
\begin{aligned}
S(t) & =S_{0} e^{Y(t)} \prod_{s \leq t}[1+\Delta Y(s)] e^{-\Delta Y(s)} \\
& =S_{0} \exp \left\{-\eta t+\int_{0}^{t} \int_{\{z \neq 0, z>-1\}} \log (1+z) N(d s, d z)\right\} .
\end{aligned}
$$

Note that if $S_{0}>a>0$, then $S(t)>0$ for all $t>0$.

Put $\tilde{S}(t)=\log S(t)$ then

$$
\tilde{S}(t)=\log S_{0}+\left\{-\eta t+\int_{0}^{t} \int_{\{z \neq 0, z>-1\}} \log (1+z) N(d s, d z)\right\} .
$$

Define

$$
\tau_{a}=\inf \{t>0 ; S(t) \leq a\},=\infty \text { if }\{\cdot\}=\phi,
$$

then

$$
\tau_{a}=\inf \{t>0 ; \tilde{S}(t) \leq \log a\}
$$

$\tau_{a}$ is the time for the economy to reach break down limit.

\subsection{Sustainable case}

We are going to investigate under what condition the system is sustainable in the sense that the system does not ruin. For ease of computations, suppose that $\left\{\nu_{i}, i<\right.$ $\infty\}$ and $\left\{\kappa_{i}, i<\infty\right\}$ are mutually independent sequences of iid random variables and they are point masses of the Poisson random measure $N(d s, d z)$. Furthermore we assume that $\nu_{0}=0$, the $\tau_{n}=\nu_{n+1}-\nu_{n}$ are exponentially distributed with mean $1 / \lambda$, and the $\kappa_{n}$ has distribution $\sigma(z) d z$. Roughly speaking, the $\nu_{n}$ and $\kappa_{n}$ mean the jump times and jump sizes of the process respectively (see Gaier, Grandis and Schachermayer (2003), pp.28 30). Note that (A.2) means that for any $n, \kappa_{n}>-1$. Then we have a lemma (c.f. Long and katayama (2002), Boikov (2003), and Fujisaki, Katayama and Ohta (2005)).

Lemma 3.1 Let $k>0$, then it follows that

$$
E\left[e^{-k \tilde{S}(t)}\right]=\exp \{-k c+t\{k \eta-\lambda(1-\Phi(k))\},
$$

where

$$
\Phi(k)=E\left[\left(1+\kappa_{i}\right)^{-k}\right]=\int(1+z)^{-k} \sigma(z) d z .
$$




\section{Proof}

$$
\begin{gathered}
E\left[e^{-k \tilde{S}(t)}\right]=E\left[\exp \left\{-k\left(c-\eta t+\int_{0}^{t} \int_{\{z \neq 0, z>-1\}} \log (1+z) N(d s, d z)\right)\right\}\right. \\
=e^{-k(c-\eta t)} E\left[\exp \left\{-k \int_{0}^{t} \int_{\{z \neq 0, z>-1\}} \log (1+z) N(d s, d z)\right\}\right] \\
=e^{-k(c-\eta t)} E\left[\exp \left\{-k \sum_{n=0}^{N(t)} \log \left(1+\kappa_{n}\right)\right\} \equiv I_{1} \times I_{2},\right.
\end{gathered}
$$

where $c=\log S_{0}$ and for each $t, N(t)$ denotes the Poisson random measure with intensity $\lambda t$, the number of jump times $\nu_{n}$ which is less than $t$.

We will calculate only the expectation part $I_{2}$. Since both sequences $\left\{\kappa_{n}\right\}$ and $\left\{\nu_{n}\right\}$ are mutually independent iid sequences,

$$
\begin{aligned}
I_{2} & =\sum_{l=0}^{\infty} E\left[\exp \left\{-k \sum_{n=0}^{l} \log \left(1+\kappa_{n}\right)\right\} ; N(t)=l\right] \\
& =\sum_{l=0}^{\infty} E\left[\left\{\prod_{i=1}^{l}\left(1+\kappa_{i}\right)\right\}^{-k}\right] \times P(N(t)=l) \\
& =\sum_{l=0}^{\infty} \prod_{i=1}^{l} E\left[\left(1+\kappa_{i}\right)^{-k}\right] \times P(N(t)=l) .
\end{aligned}
$$

Since $N(t)$ is Poisson process with intensity $\lambda t$, it follows that

$$
P(N(t)=l)=\frac{(\lambda t)^{l}}{l !} e^{-\lambda t}
$$

Then we deduce

$$
\begin{gathered}
I_{2}=\sum_{l=0}^{\infty}(\Phi(k))^{l} \frac{(\lambda t)^{l}}{l !} e^{-\lambda t} \\
=e^{-\lambda t(1-\Phi(k))}
\end{gathered}
$$

from which the assertion follows immediately. (c.q.f.d.)

Assume that there exists a $k^{*}>0$ such that

$$
k^{*} \eta-\lambda\left(1-\Phi\left(k^{*}\right)\right)=0
$$

then we have the following.

Lemma 3.2 $M^{*}(t) \equiv e^{-k^{*} \tilde{S}(t)}$ is a martingale if and only if (A.3) holds. Moreover, it can be shown that $M_{t}^{a}=M_{t \wedge \tau_{a}}^{*}$ is also martingale. 
Proof We have to show that for any $t>s, E\left[M^{*}(t) \mid \mathcal{F}_{s}\right]=M^{*}(s)$, where $\mathcal{F}_{t}$ is the filtration generated by $\left\{N_{t}\right\}$. But this follows from the fact that $M^{*}(t)$ is stationary process with independent increments and (A.3). Since $t \wedge \tau_{a}$ is bounded stopping time, the last assertion is obvious. (c.q.f.d.)

Therefore,

$$
E\left[M_{t}^{a}\right]=E\left[M_{t \wedge \tau_{a}}^{*}\right]=E\left[e^{-k^{*} \tilde{S}\left(t \wedge \tau_{a}\right)}\right]=e^{-k^{*} c}
$$

for all $t>0$. Since $\tilde{S}\left(\tau_{a}\right) \leq \log a$ on $\left\{\tau_{a} \leq t\right\}$, we deduce that

$$
P\left(\tau_{a} \leq t\right) \leq e^{-k^{*}\left(\log a_{0}-\log a\right)}
$$

for all $t>0$. Note that $c=\log S_{0}=\log a_{0}>\log a$.

An intuitive meaning of (A.3) is that average number of jumps and the sizes are small (see Remark 3.1(2)). We can summarize as follows.

Proposition 3.1.1 Assume (A.3), then

(1) $P\left(\tau_{a}<\infty\right)=\sup _{t} P\left(\tau_{a} \leq t\right) \leq e^{-k^{*}\left(\log a_{0}-\log a\right)}<1$. Therefore, in this case, the probability that exhaustion does not occur is strictly positive, in other words, the ruin probability $<1$.

(2) $P\left(\tau_{a} \leq t\right) \rightarrow 0$ as $a_{0} \rightarrow \infty$

(3) $P\left(\tau_{a} \leq t\right) \rightarrow 0$ as $a \rightarrow 0$.

The economic meaning of this proposition is that as far as average number of jumps and the sizes are small, the ruin probability of the economy within limited time is less than one. And the probability goes to zero when the initial resource stock is large or the minimum stock requirement is small.

\subsection{Ruin case}

Next we study the case when the stock process is exhaustible eventually with probability 1. Due to $(3.3), \tilde{S}(t)$ is written as follows:

$$
\tilde{S}(t)=\tilde{S}_{0}-\eta t+\tilde{J}(t)
$$

where

$$
\tilde{J}(t)=\int_{0}^{t} \int_{\mathbf{R} \backslash\{0\}} \log (1+z) N(d s, d z),
$$

For any $f \in C^{1}$, due to Ito's formula,

$$
\begin{aligned}
& f\left(\tilde{S}\left(t \wedge \tau_{a}\right)\right)-f\left(\tilde{S}_{0}\right)=\int_{0}^{t \wedge \tau_{a}}(-\eta) \partial_{x} f(\tilde{S}(s)) d s \\
& +\int_{0}^{t \wedge \tau_{a}} \int_{\mathbf{R} \backslash\{0\}}[f(\tilde{S}(s-)+\log (1+z))-f(\tilde{S}(s-))] N(d s, d z)
\end{aligned}
$$


Taking the mathematical expectation, we get

$$
\begin{aligned}
& E\left[f\left(\tilde{S}\left(t \wedge \tau_{a}\right)-f\left(\tilde{S}_{0}\right)\right]\right. \\
& =E\left[\int_{0}^{t \wedge \tau_{a}}(-\eta) \partial_{x} f(\tilde{S}(s-)) d s+\int_{0}^{t \wedge \tau_{a}} \int[f(\tilde{S}(s-)+\log (1+z))-f(\tilde{S}(s-))] \lambda d s \sigma(z) d z\right. \\
& =E\left[\int_{0}^{t \wedge \tau_{a}} L f(\tilde{S}(s-)) d s\right]
\end{aligned}
$$

where

$$
L f(x)=(-\eta) \partial_{x} f(x)+\lambda \int[f(x+\log (1+x))-f(x)] \sigma(x) d x .
$$

Assume that there exists a nondecreasing function $f \in C^{1}$ such that

$$
L f(x) \leq-1
$$

for all $x \geq \tilde{S}_{0}$. Then one deduce

$$
E\left[f\left(\tilde{S}_{t \wedge \tau_{a}}\right)-f\left(\tilde{S}_{0}\right)\right]=E\left[\int_{0}^{t \wedge \tau_{a}} L f(\tilde{S}(s)) d s\right] \leq-E\left[t \wedge \tau_{a}\right]
$$

or equivalently,

$$
E\left[t \wedge \tau_{a}\right] \leq E\left[f\left(\tilde{S}_{0}\right)-f\left(\tilde{S}_{t \wedge \tau_{a}}\right)\right]
$$

Note that the right side is bounded because

$$
\sup _{0 \leq t<\infty} E\left[f\left(\tilde{S}_{0}\right)-f\left(\tilde{S}_{t \wedge \tau_{a}}\right)\right] \leq E\left[f\left(\tilde{S}_{0}\right)-f(\log a)\right],
$$

and we have the following.

Proposition 3.2.1 Assume (A.4), then $E\left[\tau_{a}\right]$ is bounded. Moreover,

$$
P\left(\tau_{a} \geq t\right) \leq \kappa / t \rightarrow 0
$$

as $t \rightarrow \infty$, where $\kappa=f\left(\tilde{S}_{0}\right)-E\left[f\left(\tilde{S}\left(\tau_{a}\right)\right]\right.$. Therefore, in this case, the exhaustion occurs with probability 1.

It shoud be noticed that (A.3) and (A.4) are mutually inconsistent. Therefore under (A.4), it is not the case that average number of jumps and the size are small. The probability for the economy to survive eventually goes to zero under the assumption (A.4).

Remark 3.1 (1) The assumption (A.2) can be dropped and we can extend to the case where $\Delta Y(t)$ is arbitrary. But in this case $S(t)$ may take negative values so that it is not good for our case.

(2) The assumption (A.3) is equivalent to the following equation with respect to $k>0$,

$$
1-\Phi(k)=k \xi
$$


where $\xi=\eta / \lambda=\delta / \beta \lambda$. Since $\Phi(k) \geq 0, k \xi \leq 1$. Note also that it is necessary that $\Phi(k) \leq 1$ because $k \xi>0$. Since $\Phi(0)=1, k=0$ is trivial solution of Eq.(3.4). Assume that $\Phi^{\prime}(0)<0$ and choose $\xi$ such that $-\Phi^{\prime}(0)>\xi$. then it is easy to see that we can find a nontrivial solution $k>0$ of Eq.(3.4). This is equivalent to the following inequality:

$$
\int \log (1+z) \sigma(z) d z>0
$$

If the jump sizes are always positive, i.e. the density $\sigma(z)$ has compact support in $(0, \infty)$, then the above inequality is clearly satisfied, so that there is a positive solution of Eq.(3.4). However, if there arises negative jumps, then it depends on the sizes of negative jumps. Some examples are shown in the appendix in which there exists solution $k>0$.

(3) Put $f(x)=c x$ where $c$ is a positive constant. Then the assumption (A.4) means that

$$
c\left\{-\eta+\lambda \int \log (1+z) \sigma(z) d z\right\} \leq-1
$$

Suppose that

$$
-\eta+\lambda \int \log (1+z) \sigma(z) d z<0
$$

then we can choose $c>0$ so that (3.6) holds. It is obvious that this is valid if only negative jumps occur. Note also that (3.7) implies that $E[\tilde{S}(t)] \leq \log a_{0}$ for all $t$ and also $E\left[\tilde{S}\left(t \wedge \tau_{a}\right)\right] \leq \log a_{0}$. Moreover, if

$$
\int \log (1+z) \sigma(z) d z<0
$$

then (A.4) is clearly satisfied (cf.(3.5)). For example, put $\sigma(z)$ be uniformly distributed such that $\sigma(z)=1$ on $(-1 / 2,1 / 2)$, then (A.4) is fulfilled.

\section{Non-cooperative outcome}

In this section we turn to the non-cooperative scenario following Long and Katayama (2002). It should be noticed that for the existence of a Markov perfect equilibrium every agent has common knowledge on a pure jump process $J(t)$, and take it into consideration to set her optimization problem. Following the same way as Long and Katayam (2002), it turns out that there exists a Markov perfect equilibrium in which all agents play linear strategies of the form

$$
R_{j}=\mu_{j}(S)=r S
$$

and

$$
C_{j}=\theta_{j}\left(K_{j}\right)=c K_{j}
$$


Therefore the resource stock $S$ and capital stock $K_{i}$ for each agent $i$ are given by the following (cf.(2.1)):

$$
\left\{\begin{array}{l}
d K_{i}(t)=\left(R_{i}^{1-\beta} K_{i}^{\alpha}-C_{i}\right) d t \\
d S(t)=-\left(R_{i}+r(n-1) S\right) d t+d J(t),
\end{array}\right.
$$

where $r$ is a positive constant, $J(t)$ is given by (1.2) and the boundary conditions are the same as the cooperative case in $\S 2$. Moreover, suppose that each agent $i$ 's value function is given by the following.

$$
V\left(S, K_{i}\right)=\max _{C_{i}, R_{i} \geq 0} E\left[\int_{0}^{\tau_{i}}(1-\alpha)^{-1} C_{i}^{1-\alpha} e^{-\rho t} d t+g\left(S\left(\tau_{i}\right), K_{i}\left(\tau_{i}\right)\right) e^{-\rho \tau_{i}}\right],
$$

where $\tau_{i}$ is the first exit time from $D$,

$$
\tau_{i}=\inf \left\{t>0 ;\left(S(t), K_{i}(t)\right) \notin D\right\},
$$

$D=\{(S, K) ; S>a, K>0\}$ and $a$ is a positive constant. By using the same method as Long and Kayama (2002) and $\S 2$, we have the following result. Suppose that $\beta$ and the number of agents $n$ satisfies that

$$
1-n(1-\beta)>0 \text { or equivalently, } n<1 /(1-\beta) \text {. }
$$

Proposition 4.1 Assume (A.5) and that $g$ in (4.2) is given of the form

$$
g\left(S, K_{i}\right)=\hat{A} K_{i}^{1-\alpha}+\hat{B} S^{1-\beta},
$$

where $\hat{A}$ and $\hat{B}$ are positive constants. Assume also that the system is in a symmetric equilibrium, i.e.

$$
r=\left[\frac{\hat{A}(1-\alpha)}{\hat{B}}\right]^{1 / \beta} .
$$

Then the optimal consumption $C_{i}$ and the optimal resource extraction $R_{i}$ are given by

$$
\left\{\begin{array}{l}
C_{i}=\frac{\rho}{\alpha} \times K_{i} \\
R_{i}=\frac{\delta}{1-n(1-\beta)} \times S,
\end{array}\right.
$$

respectively. Moreover, the corresponding optimal trajectory of two stocks $\left(K_{i}, S\right)$ are given by the following differential equations;

$$
\left\{\begin{array}{l}
d K_{i}(t)=\left\{\left(\frac{\delta S}{1-n(1-\beta)}\right)^{1-\beta} K_{i}^{\alpha}-\left\{\frac{\rho}{\alpha}\right\} K_{i}\right\} d t \\
d S(t)=d J(t)-\left\{\frac{n \delta}{1-n(1-\beta)}\right\} S d t
\end{array}\right.
$$

with initial (boundary) conditions $K_{i}(0)=K_{0}>0$ and $S(0)=S_{0}>a>0$. 
If $1<n<1 /(1-\beta)$, then the extraction rate in the cooperative scenario is less than the non-cooperative one. It is also shown that each agent's welfare under the non-cooperative scenario is worse than the cooperative one.

Next it is interesting to calculate the ruin probability under the non-cooperative scenario. Let $\eta^{\prime}=n \delta /\{1-n(1-\beta)\}$, then $\eta<\eta^{\prime}$ if $1<n<1 /(1-\beta)$, where $\eta=\delta / \beta$. Then optimal stock process $S^{\prime}(t)$ is written as follows;

$$
S^{\prime}(t)=S_{0}+J(t)-\int_{0}^{t} \eta^{\prime} S^{\prime}(s) d s
$$

or equivalently,

$$
\begin{gathered}
d S^{\prime}(t)=S^{\prime}(t-) d Y^{\prime}(t), \\
Y^{\prime}(t)=\int_{0}^{t} \int_{\mathbf{R} \backslash\{0\}} z N(d s, d z)-\eta^{\prime} t .
\end{gathered}
$$

Put $\tilde{S}^{\prime}(t)=\log S^{\prime}(t)$ and $\tau_{a}^{\prime}=\inf \left\{t>0 ; S^{\prime}(t) \leq a\right\}$, then $\tau_{a}^{\prime}=\inf \left\{t>0 ; \tilde{S}^{\prime}(t) \leq\right.$ $\log a\}$. Since $\eta<\eta^{\prime}, \log S^{\prime}(t) \leq \log S(t)$ or equivalently, $S^{\prime}(t) \leq S(t)$. This implies that $\tau_{a}^{\prime} \leq \tau_{a}$ so that $P\left(\tau_{a}<\infty\right) \leq P\left(\tau_{a}^{\prime}<\infty\right)$. In other words, the ruin probability under non-cooperativer regime within finite time is not smaller than the one under the cooperative regime. More precisely, by using similar arguments as in $\S 3$, we can obtain the following.

Proposition 4.2 Assume that there exists a $k^{\prime *}$ such that

$$
k^{* *} \eta^{\prime}-\lambda\left(1-\Phi\left(k^{* *}\right)\right)=0
$$

then the assertion of Theorem 3.1 still holds for $\tau_{a}^{\prime}$ and $k^{\prime *}$.

As for the probability $P\left(\tau_{a}^{\prime}>t\right)$, we can proceed the same way as $\S 3$.

\section{Comparison of extraction patterns and consump- tion}

In this section we compare various extraction patterns, and discuss the role of cooperation regime and uncertainty.

The optimal resource extraction rule under cooperation without jumps:

$$
R_{h}^{C}=\frac{\rho S}{\beta n}
$$

and that of under non-cooperation without jumps:

$$
R_{h}^{N}=\frac{\rho S}{1-n(1-\beta)}
$$

by Long and Katayama (2002) . 
With jump process they are given by $(2,4)$ and $(4.3)$. Denoting $R_{h}^{C J}$ for the cooperation and $R_{h}^{N J}$ for the non-cooperation under the regimes with jump process, we have derived

$$
\begin{gathered}
R_{h}^{C J}=\frac{\delta S}{\beta n} \\
R_{h}^{N J}=\frac{\delta S}{1-n(1-\beta)}
\end{gathered}
$$

It is easily seen that

$$
R_{h}^{C J}<R_{h}^{C}<R_{h}^{N}, R_{h}^{C J}<R_{h}^{N J}<R_{h}^{N},
$$

The ordering of $R_{h}^{N J}$ and $R_{h}^{C}$ depends on the relative magnitude of effects of cooperation and uncertainty. However, we can say that agents are most conservative under cooperative regime with uncertainty and least conservative under noncooperative regime without uncertainty in terms of the resource extraction. It should be noticed that the optimal consumption rule is the same under cooperative and non-cooperative regimes as given (2.4) and (4.3). Further, it is known from Long and Katayama (2002) that the optimal level $C_{h}$ is the same as under certainty. The response for economic agents are free from uncertainty and socio-economic relationships among agents in their consumption behavior.

\section{Concluding remarks}

We have studied a differential game model of exploitation of a common property resource under unceratinty, when agents can also invest in private and productive capital. The resource extracted from a common pool is non-renewable, and the common pool is under uncertainty in the sense that it could have a sudden increase or decrease in the course of extraction. The stochastic reserve process is expressed by Lévy process.

We derived the optimal consumption and resource extraction rule and the sustainability and ruin probabilities of the stock process. One of the interesting result is that as far as average number of jumps and the sizes are small, the ruin probability of the economy within limited time is less than one. And the probability goes to zero when the initial stock is large or the minimum stock requirement is small.

The extraction rate in the cooperative regime is less than the non-cooperative regime under unceratinty. Finally it is interesting to find that ruin probability within finite time horizon under non-cooperative regime is not smaller than the one under cooperation.

\section{Appendix}

Two examples are shown here in which there exists solution $k>0$ in Eq.(3.4).

Example 1

$$
\sigma(z)= \begin{cases}0 & z<-1 / 2 \\ 2 z+1 & -1 / 2 \leq z<0 \\ e^{-(4 / 3) z} & 0 \leq z\end{cases}
$$


It is easily seen that $\Phi(0)=1, \Phi(k)$ is strictly decreasing for $0<k \leq 2$ and then it is increasing for $2<k$ and $\lim _{k \rightarrow \infty} \Phi(k)=\infty$. Therefore, if we choose $\xi$ so small that $\xi<-\Phi^{\prime}(0) \approx 0.3178$, then there exists a solution $k^{*}>0$ of Eq.(3.4).

\section{Example 2}

$$
\sigma(x)= \begin{cases}0 & z<-1 / 2 \\ 2 z+1 & -1 / 2 \leq z<0 \\ -(2 / 3) z+1 & 0 \leq z<3 / 2 \\ 0 & 3 / 2 \leq z\end{cases}
$$

If we choose $\xi$ so small that $\xi<-\Phi^{\prime}(0) \approx 0.2356$, then there exists a solution $k^{*}>0$ of Eq.(3.4). 


\section{References}

[1] Applebaum, D. Lévy Processes and Stochastic Calculus, Cambridge Univ. Press, 2004.

[2] Boikov,A.V. The Cramér-Lundberg model with stochastic premium process, Theory Probab. Appl. vol.47, No.3, pp.489-493, 2003.

[3] Fujisaki, M., Ibuki, K. and Katayama, S. Renewable common property resource and private capital accumulation Shōdai-ronshū, vol 55, No.5, pp.1-6, Kobe University of Commerce. 2004,

[4] Fujisaki, M., Katayama, S. and Ohta, H. Common property resource and private capital accumulation with random jump. In: C. Deissenberg and R. Hartl (eds), Optimal Control and Dynamic Games, pp.77-84, Springer, Dordrecht,2005.

[5] Gaier,J., Grandis,P and Schachermayer,W. Asymptotic ruin probabilities and optimal investment, The Annales of Applied Probability, vol.13, no.3, pp.10541076, 2003.

[6] Kushner, H.J. and Dupuis, P. Numerical Methods for Stochastic Control Problems in Continuous Time, Springer, 2001.

[7] Long,N.V. and Katayama,S. Common property resource and private capital accumulation. In: G.Zaccour (eds), Optimal Control and Differential Games, pp.193209, Kluwer Academic Publishers, Boston/Dordrecht/London,2002.

[8] Novikov, A. Martingales and first passage times for Ornstein-Uhlenbeck processe with a jump component, Theory Probab. Appl. vol.48, No.2, pp.288-303, 2004. 